\title{
Split Restrained Dominating Set of an Interval Graph using an Algorithm
}

\author{
A.Sudhakaraiah, PhD. \\ Assistant professor \\ Department of Mathematics \\ S.V.University,Tirupati-517502, \\ Andhra Pradesh,India.
}

\author{
E.Gnana Deepika \\ Research Scholar \\ Department of Mathematics \\ S.V.University, Tirupati-517502, \\ Andhra Pradesh,India.
}

\author{
V.Ramalatha \\ Research Scholar \\ Department of Mathematics \\ S.V.University,Tirupati-517502, \\ Andhra Pradesh,India.
}

\begin{abstract}
The various applications of the theory of Restrained domination, the most often discussed is communication network. There has been persistent in the Algorithmic aspects of interval graphs in past decades spurred much by their numerous application of an interval graphs corresponding to an interval family I. A set $D \subseteq V(G)$ is a Restrained dominating set of an Interval graph $G$, if every vertex not in $D$ is adjacent to a vertex in $D$ and to a vertex in $V-D$. In graph theory, a connected component of an undirected graph is a subgraph in which any two vertices are connected to each other by paths. For a graph $G$, if the induced subgraph of $G$ itself is a connected component then the graph $G$ is called connected otherwise $G$ is disconnected. A dominating set $D$ of $G$ is called a Split dominating set, if the induced subgraph $\langle V-D\rangle$ is disconnected. A Restrained dominating set RDS of a graph $G(V, E)$ is a Split restrained dominating set, if the induced subgraph $\langle V-R D S\rangle$ is disconnected. In this paper we present an Algorithm to find a Split Restrained dominating set of an interval graph .
\end{abstract}

\section{Key words}

Interval family, interval graph, connected graph, disconnected graph, restrained dominating set, Split restrained dominating set.

\section{INTRODUCTION}

The research of the domination in graphs has been an evergreen of the graph theory. Its basic concept is the dominating set and the domination number. The theory of domination in graphs was introduced by Ore [1] and Berge [2]. A survey on results and applications of dominating sets was presented by E.J.Cockayane and S.T.Hedetniemi [3]. In 1997 Kulli et.al introduced the concept of Split domination [4] and studied these parameters for various standard graphs and obtained the bounds for these parameters.

In general an undirected graph $G=(V, E)$ is an interval graph (IG), if the vertex set $V$ can be put into one-to-one correspondence with a set of intervals $I$ on the real line $R$, such that two vertices are adjacent in $G$, if and only if their corresponding intervals have non-empty intersection. The set $I$ is called an interval representation of $G$ and $G$ is referred to as the intersection graph $I$. Let $I=\left\{I_{1}, I_{2}, I_{3}, I_{4}, \ldots \ldots \ldots I_{n}\right\}$ be any interval family where, each $I_{i}$ is an interval on the real line and $I_{i}=\left[a_{i}, b_{i}\right]$ for $i=1,2,3,4, \ldots \ldots \ldots . . . n$. Here $a_{i}$ is called the left end point labeling and $b_{i}$ is the right end point labeling of $I_{i}$. Without loss of generality we assume that all end points of the intervals in $I$ are distinct numbers between 1 and $2 \mathrm{n}$. Two intervals $i$ and ${ }_{j}$ are said to be intersect each other if they have non empty intersection. Also we say that the intervals contain both its end points and that no two intervals share a common end point. The intervals and vertices of an interval graph are one and the same thing. The $\operatorname{graph}_{G}$ is connected and the list of sorted end point is given and the intervals in $I$ are indexed by increasing right end points, that is $b_{1}<b_{2}<b_{3}<\ldots \ldots . .<b_{n}$.

Let $G=(V, E)$ be a graph. A set $D \subseteq V(G)$ is a dominating set of $G$ if every vertex in $V / D$ is adjacent to some vertex in $D$. A set $S \subseteq V$ is a restrained dominating set (RDS) if every vertex not in $S$ is adjacent to a vertex in $S$ and to a vertex in $V-S$. Every graph has a RDS, since $S=V$ is such a set. The Restrained domination number of $\mathrm{G}$, denoted by $\gamma_{r}(G)$, is the minimum cardinality of a RDS of G. A RDS $S$ is called a $\gamma_{r}(G)$-set of $\mathrm{G}$ if $|S|=\gamma_{r}(G)$. The concept of Restrained domination was introduced by Telle and Proskurowski [5], albeit indirectly, as a vertex partitioning problem. One application of domination is that of prisoners and guards. For security, each prisoner must be seen by some guard; the concept is that of domination. However, in order to protect the rights of prisoners, we may also require that each prisoner is seen by another prisoner; the concept is that of restrained domination.

A Restrained dominating set $\operatorname{RDS}$ of $G$ is disconnected Restrained dominating set, if the induced subgraph $<V-R D S>$ is disconnected. i.e., A Restrained dominating set RDS of a graph $G(V, E)$ is a Split Restrained dominating set, if the induced subgraph $<V-R D S>$ is disconnected otherwise it is Non-split Restrained dominating set[6].

In this connection introduce the Restrained dominating set using an Algorithm [7,8,9,10]. For finding the Restrained domination [11], through an algorithm, we consider a connected Interval graph. In this Connected Interval graph the vertices are ordered by IG ordering. First of all we treat none of a vertex of $V(G)$ is a member of Restrained dominating set RDS. Then insert vertices one by one by tasting their consistency. If a vertex $v$ is dominated by at least two vertices then leave it, otherwise take the highest numbered adjacent vertex from $N[v]$ as a member of RDS if it is not adjacent to the next member of $N[v]$ or $\mathrm{v}$ is not the last vertex. 
Let us associate a new term $M_{i}(v)$ for a vertex $v \in V$, for all $i=0,1,2, \ldots ., k(k=|N(v)|)$ to each adjacent vertices of $v$ in order to IG ordering of intervals in the following way:

$M_{i}(v)=\max \left\{N[v]-\bigcup_{j=0}^{i-1} M_{i}(v)\right\}$

With $M_{0}(v)=\max \{N(v)\}$

In connection with the highest adjacent vertex of $v$, we call this $M_{i}(v)$ as the $p$ - th numbered adjacent vertex of $v$. Let $u, v \in V$. If for some

$i(i=0,1,2, \ldots . .,|N(v)|),|N(v)|-i=p$

such that $u=M_{i}(v)$, then $u$ is called the $\mathrm{p}$ - th numbered adjacent vertex of $v$.

The purpose of this paper is to find the Non-split Restrained dominating set of an Interval graph.

\section{MAIN THEOREMS}

2.1 Theorem: Let $I=\left\{i_{1}, i_{2}, \ldots . ., i_{n}\right\}$ be an n Interval family and $G$ is an Interval graph corresponding to $I$. If $i$ and $j$ are any two intervals in $I$. such that $i \in R D S$, where RDS is a Restrained Dominating Set, $j \neq 1$ and $j$ is contained in $i$, if there is at least one interval to the left of $j$ that intersect $j$ and there is no interval $k \neq i$ to the right of $j$ that intersect

$j$. Then the Restrained domination occurs in $G$ and the split restrained dominating set $\langle V-R D S\rangle$ is disconnected as $|R D S|=2$.

Proof: Let $I=\left\{i_{1}, i_{2}, \ldots . ., i_{n}\right\}$ be the given $n$ Interval family and $G$ is an interval graph corresponding to $I$. First we will find the Restrained dominatings et corresponding to $G$. If $i \& \mathrm{j}$ are any two intervals in $I$. Further by our assumption there is no interval $k>j, k \neq i$ to the right of $j$ that intersect $j$. Since $j$ is contained in $i$ implies $j<i$. Therefore $j$ is not adjacent to any vertex in the set $\{i+1, i+2,-----, n\}$ and $i \in R D S$ implies that $<V-R D S>$ does not contain $i$. This implies that clearly there is a disconnection at $j$. Thus we get the Split Restrained domination in $G$. In this procedure we also find Restrained dominating set of an interval graph towards an algorithm with an illustration as follows,

\subsection{AN ALGORITHM FOR RESTAINED}

\section{DOMINATING SET OF AN INTERVAL GRAPH}

Input: An Interval graph $G=(V, E)$ with IG ordering vertex

set $V=\{1,2, \ldots ., n\}$.

Output: Restrained Dominating Set RDS

Step 1: Set $f(j)=0, \forall j=1,2, \ldots \ldots, n$;

Step 2: Set $i=1, D=\phi$;

Step 2.1: Compute $\quad W_{i}(f)=\sum_{v \in N[i]} f(v)$

Step 2.2: If $W_{i}(f)=0$ then

Set $f\left(M_{0}(i)\right)=1, f\left(M_{1}(i)\right)=1$;

take RDS $=\left\{M_{0}(i)\right\}$.

Step 2.3: else if $W_{i}(f)=1, i$ is not the last vertex, then

Step 2.3.1: if $f\left(M_{0}(i)\right)=0, M_{0}(i)$ is adjacent to $M_{1}(i)$

RDS remains unchanged.

end if;

Step 2.3.2: otherwise if $f\left(M_{0}(i)\right)=0$,

$M_{0}(i)$ is not adjacent to $M_{1}(i)$

Set $f\left(M_{0}(i)\right)=1$

take RDS $=$ RDS $\cup\left\{M_{0}(i)\right\}$

end if;

else if $W_{i}(f)=1, i$ is the last vertex,

then RDS remains unchanged.

end if;

Step 2.3.3: if $f\left(M_{0}(i)\right)=1$,

RDS remains unchanged.

Step 2.4: else if $W_{i}(f) \geq 2$,

then RDS remains unchanged.

end if;

Step 2.5: Calculate $i=i+1$

and go to Step 2.1 and continue until the last vertex. end RDS.

Now we will find the Restrained dominating set of an interval graph with an illustration using the above algorithm as follows

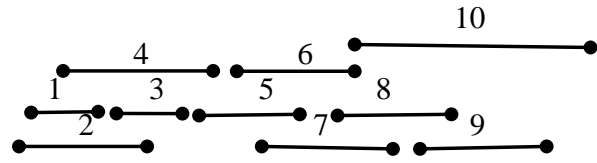

Fig.1: Interval family I

$\operatorname{nbd}[1]=\{1,2,4\}, \quad \operatorname{nbd}[2]=\{1,2,3,4\}, \operatorname{nbd}[3]=\{2,3,4\}$, nbd $[4]=\{1,2,3,4,5\}$, nbd $[5]=\{4,5,6,7\}, \quad$ nbd $[6]$ $=\{5,6,7,8,10\}$,

$\operatorname{nbd}[7]=\{5,6,7,8,10\}, \operatorname{nbd}[8]=\{6,7,8,9,10\}$

$\operatorname{nbd}[9]=\{8,9,10\}, \quad \operatorname{nbd}[10]=\{6,7,8,9,10\}$

To find the Restrained Dominating Set, we have to compute all $p$ - th numbered adjacent vertices using Table 1 . First set $f(j)=0, \forall j \in V$. In Step 2, set $i=1, \operatorname{RDS}=\phi$, that is initially RDS is empty. Step 2 repeats for $\mathrm{n}$ times. Here $n=10$, the number of vertices in the interval graph $G$.

\begin{tabular}{|l|l|l|l|l|l|l|l|l|l|l|}
\hline $\mathrm{M}_{\mathrm{i}}(\mathrm{v}) \backslash \mathrm{v}$ & 1 & 2 & 3 & 4 & 5 & 6 & 7 & 8 & 9 & 10 \\
\hline $\mathrm{M}_{0}(\mathrm{v})$ & 4 & 4 & 4 & 5 & 7 & 10 & 10 & 10 & 10 & 10 \\
& & & & & & & & & & \\
\hline $\mathrm{M}_{1}(\mathrm{v})$ & 2 & 3 & 3 & 4 & 6 & 8 & 8 & 9 & 9 & 9 \\
\hline $\mathrm{M}_{2}(\mathrm{v})$ & 1 & 2 & 2 & 3 & 5 & 7 & 7 & 8 & 8 & 8 \\
\hline $\mathrm{M}_{3}(\mathrm{v})$ & - & 1 & - & 2 & 4 & 6 & 6 & 7 & - & 7 \\
\hline $\mathrm{M}_{4}(\mathrm{v})$ & - & - & - & 1 & - & 5 & 5 & 6 & - & 6 \\
\hline
\end{tabular}

Table 1.

We follow the iterations of an illustration through the table.

Iteration (1):

For the first iteration $i=1$

$N[1]=\{1,2,4\}$

$W_{1}(f)=f(N[1])$

$W_{1}(f)=f(1)+(2)+f(4)$

$=0+0+0=0$

The first condition of if-end if is satisfied. 
Since $w_{1}(f)=0$, we find $M_{0}(1)=4, M_{1}(1)=2$

Then set $f(4)=1, \mathrm{f}(2)=1$

Also set $\operatorname{RDS}=\phi \cup\{4\} \Rightarrow \operatorname{RDS}=\{4\}$

Iteration (2):

For the second iteration $i=2$

$N[2]=\{1,2,3,4$,

$W_{2}(f)=f(N[2])$

$W_{2}(f)=f(1)+f(2)+f(3)+f(4)$

$=0+1+0+1=2$

So, in this iteration RDS could not be calculated. Hence RDS

remains same and $i$ is being increased to 3 .

Iteration (3):

For the third iteration $i=3$

$N[3]=\{2,3,4\}$

$W_{3}(f)=f(N[3])$

$W_{3}(f)=f(2)+f(3)+f(4)$

$=1+0+1=2$

In this iteration RDS remains unchanged.

The iteration number $i$ is being increased to 4 .

Iteration (4):

For the fourth iteration $i=4$

$N[4]=\{1,2,3,4,5\}$

$W_{4}(f)=f(N[4])$

$W_{4}(f)=f(1)+f(2)+f(3)+f(4)+f(5)$

$=0+1+0+1+0=2$

In this iteration RDS remains unchanged. The iteration number

$i$ is being increased to 5 .

Iteration (5):

For the fifth iteration $i=5$

$N[5]=\{4,5,6,7\}$

$W_{5}(f)=f(N[5])$

$W_{5}(f)=f(4)+f(5)+f(6)+f(7)$

$=1+0+0+0=1$

Here the Restrained domination criteria is not satisfied. The else-if condition of if-end if is satisfied. Now $f\left(M_{0}(5)\right)=f(7)=0$ and $M_{0}(5)$ is adjacent to $M_{1}(5)$. So RDS remains unchanged.The iteration number $i$ is being increased to 6 .

Iteration (6):

For the sixth iteration $i=6$

$N[6]=\{5,6,7,8,10\}$

$W_{6}(f)=f(N[6])$

$W_{6}(f)=f(5)+f(6)+f(7)+f(8)+f(10)$

$$
=0+0+0+0+0=0
$$

The first condition of if-end if is satisfied.

Since $W_{6}(f)=0$, we find $M_{0}(6)=10, M_{1}(6)=8$

Then set $f(10)=1, \mathrm{f}(8)=1$

Also set $\mathrm{RDS}=R D S \cup\{10\}$

$\Rightarrow \mathrm{RDS}=\{4\} \cup\{10\} \Rightarrow R D S=\{4,10\}$

$$
\therefore R D S=\{4,10\}
$$

The iteration number $i$ is being increased to 7 .

Iteration (7):

For the seventh iteration $i=7$

$N[7]=\{5,6,7,8,10\}$

$W_{7}(f)=f(N[7])$

$W_{7}(f)=f(5)+f(6)+f(7)+f(8)+f(10)$

$$
=0+0+0+1+1=2
$$

In this iteration RDS remains unchanged.

The iteration number $i$ is being increased to 8 .

\section{Iteration (8):}

For the eighth iteration $i=8$

$N[8]=\{6,7,8,9,10\}$

$W_{8}(f)=f(N[8])$

$W_{8}(f)=f(6)+f(7)+f(8)+f(9)+f(10)$

$$
=0+0+1+0+1=2
$$

So, in this iteration RDS remains unchanged.

The iteration number $i$ is being increased to 9 .

Iteration (9):

For the ninth iteration $i=9$

$N[9]=\{8,9,10\}$

$W_{9}(f)=f(N[9])$

$W_{9}(f)=f(8)+f(9)+f(10)$

$$
=1+0+1=2
$$

In this iteration RDS could not be calculated. Hence RDS remains unchanged and $i$ is being increased to 10 .

Iteration (10):

For the tenth iteration $i=10$

$N[10]=\{6,7,8,9,10\}$

$W_{10}(f)=f(N[10])$

$W_{10}(f)=f(6)+f(7)+f(8)+f(9)+f(10)$

$=0+0+1+0+1=2$

So, in this iteration RDS remains unchanged.

$\therefore$ RDS $=\{4,10\}$

$|R D S|=$ The cardinality of $\mathrm{RDS}=2$.

Thus we get the Spilt Restrained dominating set $\langle V-R D S\rangle$ as follows,

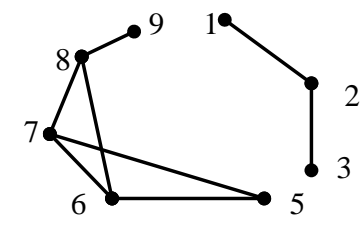

Fig.2: Vertex induced subgraph $<V-R D S>-$ Disconnected graph from $\mathbf{G}$

2.3 THEOREM: Let $\mathrm{G}$ be an Interval graph corresponding to an $\mathrm{n}$ Interval family $I=\left\{i_{1}, i_{2}, \ldots ., i_{n}\right\}$. If $i$ and $j$ are any two intervals in $I$. such that $j$ is contained in $i$ and there is an interval $k \neq i$ such that $k \in R D S$, that intersects $i$ and there is no other interval to the right of $k$ that intersect $j$, then Restrained domination occurs in $G$ and the Split Restrained dominating set $<V-R D S>$ is disconnected as $|R D S|=3$.

Proof: Let $I=\left\{i_{1}, i_{2}, \ldots . ., i_{n}\right\}$ be the given n Interval family and $G$ is an interval graph corresponding to $I$. Let $i$ and $j$ be any two intervals of $I$. such that $j$ is contained in $i$ and there is an interval $k \neq i$ such that $k \in R D S$, that intersects $i$ and $j$ also. Since $k \in R D S,<V-R D S>$ does not contain $k$. Further there is a connection for the intervals $i$ and $j$ to the interval $k$ and there is no other interval to the right of $i$ that intersect $j$, hence there will be a disconnection in $\langle V-R D S\rangle$. Since both the intervals $i$ and $j$ intersects together, there is no isolated vertex in $\langle V-R D S\rangle$. Therefore we get the Split Restrained domination in $G$. 
Next we will find the Restrained dominating set as follows from an interval family using the Algorithm as explained in section 2.2.

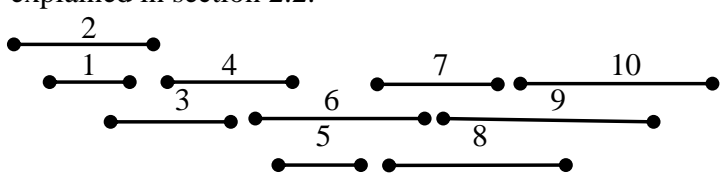

Fig.3: Interval Family $I$

$\operatorname{nbd}[1]=\{1,2,3\}, \operatorname{nbd}[2]=\{1,2,3\}, \operatorname{nbd}[3]=\{1,2,3,4\}$, $\operatorname{nbd}[4]=\{3,4,5,6\}, \operatorname{nbd}[5]=\{4,5,6\}, \operatorname{nbd}[6]=\{4,5,6,7,8\}$, $\operatorname{nbd}[7]=\{6,7,8,9\}, \operatorname{nbd}[8]=\{6,7,8,9,10\}$,

$\operatorname{nbd}[9]=\{7,8,9,10\}, \operatorname{nbd}[10]=\{8,9,10\}$

To find the Restrained Dominating Set, we have to compute all p-- th numbered adjacent vertices from the following table,

\begin{tabular}{|l|l|l|l|l|l|l|l|l|l|l|}
\hline $\mathrm{M}_{\mathrm{i}}(\mathrm{v}) \backslash \mathrm{v}$ & 1 & 2 & 3 & 4 & 5 & 6 & 7 & 8 & 9 & 10 \\
\hline $\mathrm{M}_{0}(\mathrm{v})$ & 3 & 3 & 4 & 6 & 6 & 8 & 9 & 10 & 10 & 10 \\
\hline $\mathrm{M}_{1}(\mathrm{v})$ & 2 & 2 & 3 & 5 & 5 & 7 & 8 & 9 & 9 & 9 \\
\hline $\mathrm{M}_{2}(\mathrm{v})$ & 1 & 1 & 2 & 4 & 4 & 6 & 7 & 8 & 8 & 8 \\
\hline $\mathrm{M}_{3}(\mathrm{v})$ & - & - & 1 & 3 & - & 5 & 6 & 7 & 7 & - \\
\hline $\mathrm{M}_{4}(\mathrm{v})$ & - & - & - & - & - & 4 & - & 6 & - & - \\
\hline
\end{tabular}

Table 2

First set $f(j)=0, \forall j \in V$. In Step 2, set $i=1$, $\operatorname{RDS}=\phi$, that is initially RDS is empty. Step 2 repeats for $n$ times. Here $n=10$, the number of vertices in the interval graph $G$. We follow the iterations of an illustration through the table.

\section{Iteration (1):}

For the first iteration $i=1$

$N[1]=\{1,2,3\}$

$W_{1}(f)=f(N[1])$

$W_{1}(f)=f(1)+(2)+f(3)$

$$
=0+0+0=0
$$

The first condition of if-end if is satisfied.

Since $w_{1}(f)=0$, we find $M_{0}(1)=3, M_{1}(1)=2$

Then set $f(3)=1, \mathrm{f}(2)=1$

Also set $\mathrm{RDS}=\phi \cup\{3\}$

$\Rightarrow \mathrm{RDS}=\{3\}$

\section{Iteration (2):}

For the second iteration $i=2$

$N[2]=\{1,2,3\}$

$W_{2}(f)=f(N[2])$

$W_{2}(f)=f(1)+f(2)+f(3)$

$$
=0+1+1=2
$$

So, in this iteration RDS could not be calculated. Hence RDS remains same and $i$ is being increased to 3 .

\section{Iteration (3):}

For the third iteration $i=3$

$N[3]=\{1,2,3,4\}$

$W_{3}(f)=f(N[3])$

$W_{3}(f)=f(1)+f(2)+f(3)+f(4)$

$$
=0+1+1+0=2
$$

In this iteration RDS remains unchanged. The iteration number

$i$ is being increased to 4 .

Iteration (4):

For the fourth iteration $i=4$
$N[4]=\{3,4,5,6\}$

$W_{4}(f)=f(N[4])$

$W_{4}(f)=f(3)+f(4)+f(5)+f(6)$

$$
=1+0+0+0=1
$$

Here the Restrained domination criteria is not satisfied. The else-if condition of if-end if is satisfied. Now $f\left(M_{0}(4)\right)=f(6)=0$ and $M_{0}(4)$ is adjacent to $M_{1}(4)$. So RDS remains unchanged.

The iteration number $i$ is being increased to 5 .

Iteration (5):

For the fifth iteration $i=5$

$N[5]=\{4,5,6\}$

$W_{5}(f)=f(N[5])$

$W_{5}(f)=f(4)+f(5)+f(6)$

$$
=0+0+0=0
$$

The first condition of if-end if is satisfied.

Since $W_{5}(f)=0$, we find $M_{0}(5)=6, M_{1}(5)=5$

Then set $f(6)=1, \mathrm{f}(5)=1$

Also set $\quad \mathrm{RDS}=R D S \cup\{6\}$

$\Rightarrow \operatorname{RDS}=\{3\} \cup\{6\} \Rightarrow \operatorname{RDS}=\{3,6\}$

The iteration number $i$ is being increased to 6 .

Iteration (6):

For the sixth iteration $i=6$

$N[6]=\{4,5,6,7,8\}$

$W_{6}(f)=f(N[6])$

$W_{6}(f)=f(4)+f(5)+f(6)+f(7)+f(8)$

$=0+1+1+0+0=2$

In this iteration RDS remains unchanged. The iteration number $i$ is being increased to 7 .

\section{Iteration (7):}

For the seventh iteration $i=7$

$N[7]=\{6,7,8,9\}$

$W_{7}(f)=f(N[7])$

$W_{7}(f)=f(6)+f(7)+f(8)+f(9)$

$$
=1+0+0+0=1
$$

Here the Restrained domination criteria is not satisfied. The else-if condition of if-end if is satisfied. Now $f\left(M_{0}(7)\right)=f(9)=0$ and $M_{0}(7)$ is adjacent to $M_{1}(7)$. So RDS remains unchanged.

The iteration number $i$ is being increased to 8 .

Iteration (8):

For the eighth iteration $i=8$

$N[8]=\{6,7,8,9,10\}$

$W_{8}(f)=f(N[8])$

$W_{8}(f)=f(6)+f(7)+f(8)+f(9)+f(10)$

$$
=1+0+0+0+0=1
$$

Here also the Restrained domination criteria is not satisfied. The else-if condition of if-end if is satisfied. Now $f\left(M_{0}(8)\right)=f(10)=0$ and $M_{0}(8)$ is adjacent to $M_{1}(8)$. So RDS remains unchanged.

The iteration number $i$ is being increased to 9 .

Iteration (9):

For the ninth iteration $i=9$

$N[9]=\{7,8,9,10\}$

$W_{9}(f)=f(N[9])$

$W_{9}(f)=f(7)+f(8)+f(9)+f(10)$

$=0+0+0+0=0$ 
The first condition of if-end if is satisfied. Since $W_{9}(f)=0$, we find $M_{0}(9)=10, M_{1}(9)=9$

Then set $f(10)=1, \mathrm{f}(9)=1$

Also set $\mathrm{RDS}=\operatorname{RDS} \cup\{10\}$

$\Rightarrow \mathrm{RDS}=\{3,6\} \cup\{10\} \Rightarrow \operatorname{RDS}=\{3,6,10\}$

Iteration (10):

For the tenth iteration $i=10$

$N[10]=\{8,9,10\}$

$W_{10}(f)=f(N[10])$

$W_{10}(f)=f(8)+f(9)+f(10)$

$$
=0+1+1
$$$$
=2
$$

So, in this iteration RDS remains unchanged.

$\therefore \operatorname{RDS}=\{3,6,10\}$

$|R D S|=$ The cardinality of $\mathrm{RDS}=3$.

Thus we get the Spilt restrained dominating set $<V-R D S>$ as follows,

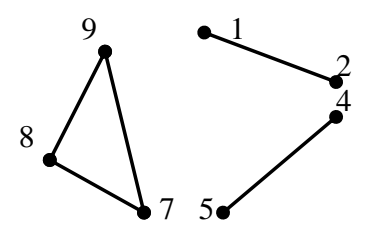

Fig.4: Vertex induced subgraph $<V-R D S>$ - Disconnected graph from $G$

2.4 THEOREM: Let us consider an $\mathrm{n}$ interval family $I=\left\{i_{1}, i_{2}, \ldots . ., i_{n}\right\}$ and $G$ be an interval graph of $I$. If $i, j, k$ are any three consecutive intervals such that $i<j<k$ and $k \in R D S, i$ intersect $j, j$ intersect $k$ and $i$ intersect $k$ and also there is no other interval to the right of $k$ that intersect $k$ but not $i$ and $j$ Then Restrained domination occurs in $G$ and the Split restrained dominating set $\langle V-R D S\rangle$ is disconnected as $|R D S|=3$.

Proof: Let $I=\left\{i_{1}, i_{2}, \ldots . ., i_{n}\right\}$ be an $\mathrm{n}$ interval family and ${ }_{G}$ be an interval graph of $I$. Let $i, j, k$ be three consecutive intervals satisfies the hypothesis. Suppose $k \in R D S$ for $i<j<k, i$ intersects $k$, there is no other interval to the right of $k$ that intersect $k$ but not $i$ and $j$ provided there is an $m \in I, m>k$ such that mintersect $i$ and $j$ also. If such an mexists, still there is a connection with $i$ and $j$ to $m$ in $\langle V-R D S\rangle$. This is a contradiction to the hypothesis. So such an $m$ does not exists. Hence we get Split Restrained domination and $<V-R D S>$ is disconnected.

Now we will find the Restrained dominating set using the Algorithm as given in Section 2.2 as follows. For this consider the following interval family,

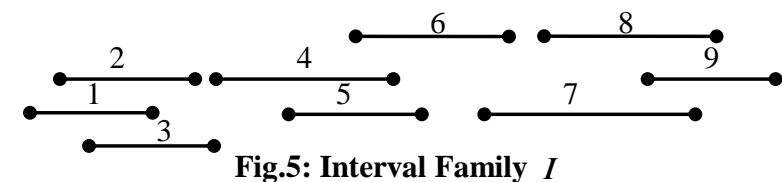

$\operatorname{nbd}[1]=\{1,2,3\}, \operatorname{nbd}[2]=\{1,2,3\}, \operatorname{nbd}[3]=\{1,2,3,4\}$, $\operatorname{nbd}[4]=\{3,4,5,6\}, \operatorname{nbd}[5]=\{4,5,6\}, \operatorname{nbd}[6]=\{4,5,6,7\}$, $\operatorname{nbd}[7]=\{6,7,8,9\}, \operatorname{nbd}[8]=\{7,8,9\}, \operatorname{nbd}[9]=\{7,8,9\}$.

To find the Restrained Dominating Set, we have to compute all p- th numbered adjacent vertices using Table 2 . First set $f(j)=0, \forall j \in V$. In Step 2, set $i=1$,

RDS $=\phi$, that is initially RDS is empty. Step 2 repeats for $\mathrm{n}$ times. Here $n=9$, the number of vertices in the interval graph $G$.

\begin{tabular}{|l|l|l|l|l|l|l|l|l|l|}
\hline $\mathrm{M}_{\mathrm{i}}(\mathrm{v}) \backslash \mathrm{v}$ & 1 & 2 & 3 & 4 & 5 & 6 & 7 & 8 & 9 \\
\hline $\mathrm{M}_{0}(\mathrm{v})$ & 3 & 3 & 4 & 6 & 6 & 7 & 9 & 9 & 9 \\
\hline $\mathrm{M}_{1}(\mathrm{v})$ & 2 & 2 & 3 & 5 & 5 & 6 & 8 & 8 & 8 \\
\hline $\mathrm{M}_{2}(\mathrm{v})$ & 1 & 1 & 2 & 4 & 4 & 5 & 7 & 7 & 7 \\
\hline $\mathrm{M}_{3}(\mathrm{v})$ & - & - & 1 & 3 & - & 4 & 6 & - & - \\
\hline
\end{tabular}

\section{Table 3}

As follows iterations,

Iteration (1):

For the first iteration $i=1$

$N[1]=\{1,2,3\}$

$W_{1}(f)=f(N[1])$

$W_{1}(f)=f(1)+(2)+f(3)$.

$$
=0+0+0=0 \text {. }
$$

The first condition of if-end if is satisfied. Since $w_{1}(f)=0$, we find $M_{0}(1)=3, M_{1}(1)=2$

Then set $f(3)=1, \mathrm{f}(2)=1$

Also set $\operatorname{RDS}=\phi \cup\{3\} \Rightarrow \operatorname{RDS}=\{3\}$

Iteration (2):

For the second iteration $i=2$

$N[2]=\{1,2,3\}$

$W_{2}(f)=f(N[2])$

$W_{2}(f)=f(1)+f(2)+f(3)$

$$
=0+1+1=2
$$

So, in this iteration RDS could not be calculated. Hence RDS remains same and $i$ is being increased to 3 .

\section{Iteration (3):}

For the third iteration $i=3$

$N[3]=\{1,2,3,4\}$

$W_{3}(f)=f(N[3])$

$W_{3}(f)=f(1)+f(2)+f(3)+f(4)$

$$
=0+1+1+0=2
$$

In this iteration RDS remains unchanged. The iteration number

$i$ is being increased to 4

Iteration (4):

For the fourth iteration $i=4$

$N[4]=\{3,4,5,6\}$

$W_{4}(f)=f(N[4])$

$W_{4}(f)=f(3)+f(4)+f(5)+f(6)$

$$
=1+0+080=1
$$

Here the Restrained domination criteria is not satisfied. The else-if condition of if-end if is satisfied. Now $f\left(M_{0}(4)\right)=f(6)=0$ and $M_{0}(4)$ is adjacent to $M_{1}(4)$. So RDS remains unchanged. The iteration number $i$ is being increased to 5 . 


\section{Iteration (5):}

For the fifth iteration $i=5$

$N[5]=\{4,5,6\}$

$W_{5}(f)=f(N[5])$

$W_{5}(f)=f(4)+f(5)+f(6)$

$$
=0+0+0=0
$$

The first condition of if-end if is satisfied.

Since $W_{5}(f)=0$, we find $M_{0}(5)=6, M_{1}(5)=5$

Then set $f(6)=1, \mathrm{f}(5)=1$

Also set $\quad \mathrm{RDS}=R D S \cup\{6\}$

$\Rightarrow \mathrm{RDS}=\{3\} \cup\{6\} \Rightarrow \mathrm{RDS}=\{3,6\}$

The iteration number $i$ is being increased to 6 .

Iteration (6):

For the sixth iteration $i=6$

$N[6]=\{4,5,6,7\}$

$W_{6}(f)=f(N[6])$

$W_{6}(f)=f(4)+f(5)+f(6)+f(7)$

$$
=0+1+1+0=2
$$

In this iteration RDS remains unchanged. The iteration number

$i$ is being increased to 7 .

Iteration (7):

For the seventh iteration $i=7$

$N[7]=\{6,7,8,9\}$

$W_{7}(f)=f(N[7])$

$W_{7}(f)=f(6)+f(7)+f(8)+f(9)$

$$
=1+0+0+0=1
$$

Here the Restrained domination criteria is not satisfied. The else-if condition of if-end if is satisfied. Now $f\left(M_{0}(7)\right)=f(9)=0$ and $M_{0}(7)$ is adjacent to $M_{1}(7)$. So RDS remains unchanged.

The iteration number $i$ is being increased to 8 .

Iteration (8):

For the eighth iteration $i=8$

$N[8]=\{7,8,9\}$

$W_{8}(f)=f(N[8])$

$W_{8}(f)=f(7)+f(8)+f(9)$

$$
=0+0+0=0
$$

The first condition of if-end if is satisfied.

Since $W_{8}(f)=0$, we find $M_{0}(8)=9, M_{1}(8)=8$

Then set $f(9)=1, \mathrm{f}(8)=1$

Also set $\quad \mathrm{RDS}=R D S \cup\{9\}$

$\Rightarrow \operatorname{RDS}=\{3,6\} \cup\{9\} \Rightarrow \operatorname{RDS}=\{3,6,9\}$

The iteration number $i$ is being increased to 9 .

Iteration (9):

For the ninth iteration $i=9$

$N[9]=\{7,8,9\}$

$W_{9}(f)=f(N[9])$

$W_{9}(f)=f(7)+f(8)+f(9)$

$$
=0+1+1=2
$$

In this iteration RDS remains unchanged. Here 9 is the last vertex, then the interation processs will end.

$\therefore \operatorname{RDS}=\{3,6,9\}$

$|R D S|=$ The cardinality of $\operatorname{RDS}=3$.

Thus we get the Spilt restrained dominating set $\langle V-R D S\rangle$ as follows,

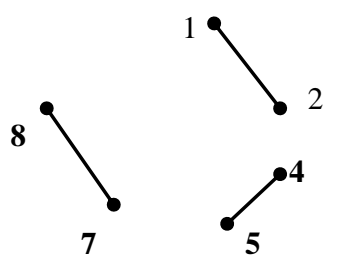

Fig.6: Vertex induced subgraph $<V-R D S>$ - disconnected graph from $\mathbf{G}$

\section{ACKNOWLEDGMENT}

The authors are greatful to the referees for their valuable comments wich have lead to improvements in the presentation of the paper. This research was supported in part by B.Maheswari, Sri Padmavathi Women's University,Tirupati, Andhra Pradesh, Tirupati.

\section{REFERENCES}

[1] O.Ore, Theory of Graph, Amer, Math.Soc.Colloq.Publ.38, Providence (1962), P.206

[2] C.Berge, Graphs and Hyperactive graphs,North Holland, Amsterdram in graphs, Networks, Vol.10 1980), 211-215.

[3] E.J.Cockayne, S.T.Hedetniemi, Towards a theory of domination in graphs, Networks, Vol.7(1977), 247-261.

[4] V.R.Kulli, B.Janakiram, The Split domination number of a graph, Graph Theory, Notes of New York, Vol.XXXII, 16-19(1997).

[5] J.A.Telle and A.Proskurowski, Algorithms for vertex portioning problems on partial k-trees. Siam J. Discrete Math. 10 (1997) 529-550.

[6] Dr.A.Sudhakaraiah, E.Gnana Deepika, V.Rama Latha, T.Venkateswarulu, Non-Split Restrained Dominating Set Of An Interval Graph Using An Algorith, Internation Journal of Advancements in Research \& Technology ISSN 2278-7763, Volume 1, Issue 2, July-2012.

[7] M.Pal, S.Mondal, D.Bera and T.K. Pal, An optimal parallel algorithm for computing cutvertices and blocks on interval graphs, International Journal of Computer Mathematics, 75 (2000) 59-70.

[8] M.C. Golumbic, Algorithmic Graph Theory and Perfect Graphs, Academic Press, New York, 1980.

[9] Tarasankar Pramanik, Sukumar Mondal and Madhumangal Pal, Minimum 2-tuple dominating set of an interval graph.

[10] Dr.A.Sudhakaraiah, E.Gnana Deepika, V.Rama Latha, To ind a 2-tuple dominating set of an induced subgraph of a non-split dominating set of an interval graph using a algorithm, International Journal of Engineering Research and Technology, ISSN:2278-0181, Vol. 1 Issue 3, May2012

[11] G.S.Domke, J.H. Hattingh, M.A.Henning, and L.R.Markus, Restrained domination in graphs with minimum degree two. J.Combin.Math.Combin.Comput.35 (2000) 239-254. 